\title{
Vacuum fluctuations and the thermodynamics of chiral models
}

\author{
V. Skokov, ${ }^{1}$ B. Friman, ${ }^{1}$ E. Nakano, ${ }^{1,2}$ K. Redlich,,${ }^{3,4}$ and B.-J. Schaefer ${ }^{5}$ \\ ${ }^{1}$ GSI Helmholtzzentrum für Schwerionenforschung, D-64291 Darmstadt, Germany \\ ${ }^{2}$ Physics Division, Faculty of Science, Kochi University, Kochi 780-8520, Japan \\ ${ }^{3}$ Institute of Theoretical Physics, University of Wroclaw, PL-50204 Wroctaw, Poland \\ ${ }^{4}$ Theory Division, CERN, CH-1211 Geneva 23, Switzerland \\ ${ }^{5}$ Institut für Physik, Karl-Franzens-Universität, A-8010 Graz, Austria
}

(Dated: November 9, 2018)

\begin{abstract}
We consider the thermodynamics of chiral models in the mean-field approximation and discuss the relevance of the (frequently omitted) fermion vacuum loop. Within the chiral quark-meson model and its Polyakov loop extended version, we show that the fermion vacuum fluctuations can change the order of the phase transition in the chiral limit and strongly influence physical observables. We compute the temperature-dependent effective potential and baryon number susceptibilities in these models, with and without the vacuum term, and explore the cutoff and the pion mass dependence of the susceptibilities. Finally, in the renormalized model the divergent vacuum contribution is removed using the dimensional regularization.

PACS numbers: 24.85.+p, 21.65.-f, 25.75.-q, 24.60.-k
\end{abstract}

\section{INTRODUCTION}

The critical properties of strongly interacting matter near the chiral transition at finite temperature and density are studied in first principle calculations using lattice simulations of QCD [1]. A complementary approach is offered by effective chiral models [2-11], like the Nambu-Jona-Lasinio (NJL) 3] and the chiral quarkmeson (QM) [11] models as well as the corresponding extended versions, which include the interaction of quarks with a uniform temporal gluon field. The latter, the Polyakov loop extended Nambu-Jona-Lasinio (PNJL) [5, 6] and quark-meson (PQM) 7, 8] models reproduce prominent features of QCD thermodynamics, obtained in lattice simulations. In particular, both models exhibit a chiral as well as a deconfinement transition.

In this paper, the consistency of mean-field calculations of many-body systems within chiral effective models like the NJL/PNJL and QM/PQM models is addressed. In particular, we discuss the role of the lowest order fermion vacuum contribution. In the NJL/PNJL model, this term is responsible for the dynamical breaking of the chiral symmetry in vacuum and is therefore necessarily taken into account. On the other hand, in the QM/PQM model, the spontaneous breaking of the chiral symmetry is due to the meson potential. Hence, for qualitative considerations, the fermion vacuum loop is frequently omitted 10,12 17.

We show that a mean-field approximation, where the fermion vacuum term is neglected, commonly referred to as the no-sea approximation, leads to a distortion of the critical behavior at the chiral transition. In particular, in this approximation the order of the transition in the chiral limit is always first order. In contrast, when this term is included, the transition is of first or second order, depending on the choice of coupling constants and on the baryon density.

Differences in the thermodynamics between the NJL and QM models found in the literature can be attributed to the use of the no-sea approximation in the QM model. For instance, in this approximation, the adiabatic trajectories obtained in the QM model exhibit a kink at the chiral crossover transition, while in the NJL model they are smooth everywhere [10]. This effect is a trace of the underlying first-order phase transition of the QM model in the chiral limit, when fermionic vacuum fluctuations are neglected [18]. Moreover, the omission of fermion vacuum fluctuations can result in a violation of the semi-positivity of the spectral function as well as in a violation of detailed balance [19]. The vacuum fermion fluctuations were also shown in the PQM model to considerably affect the structure of the phase diagram in the presence of an external magnetic field [20].

In this paper we demonstrate the importance of fermion vacuum fluctuations for a consistent formulation of the thermodynamics of the QM and PQM models in the mean-field approximation. We show that, in order to reproduce the second-order chiral phase transition expected for two flavor QCD within these models 21], it is necessary to take the fermion vacuum term in the thermodynamic potential into account and to perform a proper regularization of the divergence. Furthermore, we also show that when the chiral symmetry is explicitly broken, the thermodynamics is strongly affected by the no-sea approximation. Finally, we remove the infinities of the vacuum term using the dimensional regularization.

\section{THERMODYNAMICS OF THE QUARK-MESON MODEL}

The chiral QM model is used as an effective realization of the low-energy sector of QCD. The replacement of the $S U\left(N_{c}\right)$ local gauge invariance of QCD by a global symmetry in the QM model eliminates the possibility to address confinement within this model. However, as re- 
cently shown, the confining properties of QCD can be effectively accounted for by introducing the Polyakov loop in the chiral quark-meson model [7], in complete analogy to the PNJL model [5]. Consequently, the PQM model is an effective model of both the chiral and confining properties of QCD.

The Lagrangian of the PQM model reads

$$
\begin{aligned}
\mathcal{L}= & \bar{q}\left[i \gamma^{\mu} D_{\mu}-g\left(\sigma+i \gamma_{5} \vec{\tau} \cdot \vec{\pi}\right)\right] q+\frac{1}{2}\left(\partial_{\mu} \sigma\right)^{2}+\frac{1}{2}\left(\partial_{\mu} \vec{\pi}\right)^{2} \\
& -U(\sigma, \vec{\pi})-\mathcal{U}\left(\ell, \ell^{*}\right)
\end{aligned}
$$

where $\mathcal{U}\left(\ell, \ell^{*}\right)$ is an effective potential of the gluon field expressed in terms of the thermal expectation values of the color trace of the Polyakov loop and its conjugate,

$$
\ell=\frac{1}{N_{c}}\left\langle\operatorname{Tr}_{c} L(\vec{x})\right\rangle, \quad \ell^{*}=\frac{1}{N_{c}}\left\langle\operatorname{Tr}_{c} L^{\dagger}(\vec{x})\right\rangle,
$$

with

$$
L(\vec{x})=\mathcal{P} \exp \left(i \int_{0}^{\beta} d \tau A_{4}(\vec{x}, \tau)\right)
$$

Here $\mathcal{P}$ stands for the path ordering, $\beta=1 / T$ and $A_{4}=$ $i A_{0}$. We use the short-hand notation $A_{\mu}=g \lambda^{a} A_{\mu}^{a} / 2$.

The $O(4)$ representation of the meson fields is $\phi=$ $(\sigma, \vec{\pi})$ and the corresponding $S U(2)_{L} \otimes S U(2)_{R}$ chiral representation is given by $\sigma+i \gamma_{5} \vec{\tau} \cdot \vec{\pi}$. This implies that there are $N_{f}^{2}=4$ mesonic degrees of freedom coupled to $N_{f}=2$ flavors of constituent quarks. The coupling between the effective gluon field and quarks is implemented through the covariant derivative

$$
D_{\mu}=\partial_{\mu}-i A_{\mu}
$$

with $A_{\mu}=\delta_{\mu 0} A_{0}$. The purely mesonic potential of the model reads

$$
U(\sigma, \vec{\pi})=\frac{\lambda}{4}\left(\sigma^{2}+\vec{\pi}^{2}-v^{2}\right)^{2}-h \sigma,
$$

while the Polyakov loop potential is parameterized by a $Z(3)$ invariant form:

$$
\frac{\mathcal{U}\left(T ; \ell, \ell^{*}\right)}{T^{4}}=-\frac{b_{2}(T)}{2} \ell^{*} \ell-\frac{b_{3}}{6}\left(\ell^{3}+\ell^{* 3}\right)+\frac{b_{4}}{4}\left(\ell^{*} \ell\right)^{2} .
$$

The parameters,

$$
b_{2}(T)=a_{0}+a_{1}\left(\frac{T_{0}}{T}\right)+a_{2}\left(\frac{T_{0}}{T}\right)^{2}+a_{3}\left(\frac{T_{0}}{T}\right)^{3},
$$

where $a_{0}=6.75, a_{1}=-1.95, a_{2}=2.625, a_{3}=-7.44$, $b_{3}=0.75$ and $b_{4}=7.5$ are chosen to reproduce the equation of state obtained on the lattice for pure $\mathrm{SU}(3)$ gauge theory [6], which yields a first-order phase transition at the temperature $T_{0}=270 \mathrm{MeV}$.

The thermodynamic potential of the PQM model is in the mean-field approximation [7]

$$
\Omega_{\mathrm{MF}}\left(T, \mu ;\langle\sigma\rangle, \ell, \ell^{*}\right)=\mathcal{U}\left(T ; \ell, \ell^{*}\right)+U(\langle\sigma\rangle,\langle\vec{\pi}\rangle=\overrightarrow{0})+\Omega_{q \bar{q}}\left(T, \mu ;\langle\sigma\rangle, \ell, \ell^{*}\right)
$$

The quark contribution, with dynamical mass $m_{q}=g\langle\sigma\rangle$ and energy $E_{q}=\sqrt{\vec{p}^{2}+m_{q}^{2}}$, is given by

$$
\Omega_{q \bar{q}}\left(T, \mu ;\langle\sigma\rangle, \ell, \ell^{*}\right)=-2 N_{f} \int \frac{d^{3} p}{(2 \pi)^{3}}\left\{N_{c} E_{q} \theta\left(\Lambda^{2}-\vec{p}^{2}\right)+T \ln g^{(+)}\left(T, \mu ;\langle\sigma\rangle, \ell, \ell^{*}\right)+T \ln g^{(-)}\left(T, \mu ;\langle\sigma\rangle, \ell, \ell^{*}\right)\right\}
$$

where

$$
\begin{aligned}
& g^{(+)}\left(T, \mu ;\langle\sigma\rangle, \ell, \ell^{*}\right)=1+3 \ell e^{-\left(E_{q}-\mu\right) / T}+3 \ell^{*} e^{-2\left(E_{q}-\mu\right) / T}+e^{-3\left(E_{q}-\mu\right) / T}, \\
& g^{(-)}\left(T, \mu ;\langle\sigma\rangle, \ell, \ell^{*}\right)=g^{(+)}\left(T,-\mu ;\langle\sigma\rangle, \ell^{*}, \ell\right) .
\end{aligned}
$$

The first term in Eq. (9) is the fermion vacuum contribution, regularized by the ultraviolet cutoff $\Lambda$.

The equations of motion for the mean fields $\langle\sigma\rangle, \ell$ and $\ell^{*}$ follow from the stationarity conditions

$$
\frac{\partial \Omega_{\mathrm{MF}}}{\partial \sigma}=\frac{\partial \Omega_{\mathrm{MF}}}{\partial \ell}=\frac{\partial \Omega_{\mathrm{MF}}}{\partial \ell^{*}}=0,
$$

which yield the temperature and chemical potential dependence of the chiral $\langle\sigma\rangle(T, \mu)$ as well as of the Polyakov loops $\ell(T, \mu)$ and $\ell^{*}(T, \mu)$ order parameters.
The parameters $h, g, \lambda$ and $v$ are specified by requiring that the following vacuum properties be reproduced: the chiral condensate is fixed to $\langle\sigma\rangle(0,0)=f_{\pi}$, the pion mass to $m_{\pi}^{2}=h / f_{\pi}$, the constituent mass of quarks to $m_{q}^{0}=$ $g f_{\pi}$ and the sigma mass to

$$
m_{\sigma}^{2}=\frac{\partial^{2} \Omega_{\mathrm{MF}}\left(0,0 ; f_{\pi}, \ell, \ell^{*}\right)}{\partial \sigma^{2}}
$$

at the potential minimum. In the following we use the 
values $m_{\pi}=138 \mathrm{MeV}, m_{\sigma}=700 \mathrm{MeV}, m_{q}^{0}=335 \mathrm{MeV}$ and $f_{\pi}=93 \mathrm{MeV}$.

The results in the chiral limit are obtained with the same parameters except for the symmetry breaking term $h$, which is set to zero. Thus, we ignore any influence of the pion mass on the other parameters, like e.g. $f_{\pi}$ [22]. For the problem at hand, such shifts are unimportant.

The thermodynamic potential in the QM model is obtained from Eq. (8) by setting $\ell=\ell^{*}=1$ and ignoring the constant contribution from the Polyakov loop potential. In this case the functions $g^{( \pm)}$reduce to

$$
g^{( \pm)}\left(T, \mu ;\langle\sigma\rangle, \ell=1, \ell^{*}=1\right)=\left[1-n_{F}(T, \pm \mu ;\langle\sigma\rangle)\right]^{-3},
$$

where

$$
n_{F}(T, \pm \mu ;\langle\sigma\rangle)=\left[\exp \left(\frac{E_{q} \pm \mu}{T}\right)+1\right]^{-1}
$$

is the Fermi-Dirac distribution function. In the following, we do not consider fluctuations of the $\sigma$ field. Hence, we simplify the notation by dropping the bra-ket notation. Thus, from this point on, $\sigma \equiv\langle\sigma\rangle$ denotes a uniform classical field.

\section{LANDAU EFFECTIVE THEORY}

The mean-field thermodynamic potential (8) can be used to explore the influence of fermion vacuum fluctuations on the critical and thermal properties of a system of quarks interacting with the meson and gluon mean fields. In this section we explore the role of the fermion vacuum term in an effective Landau theory for the QM model with and without the fermion vacuum term. Thus, for the moment we neglect the Polyakov loop potential and its coupling to the quarks. The conclusions of this section are, however, directly applicable also to the Polyakov loop extension of the QM model.

\section{A. Effective potential without fermion vacuum fluctuations}

The contribution of the fermion vacuum term to the effective potential (9) can be eliminated by setting the cutoff parameter $\Lambda$ to zero. In the chiral limit, i.e., for $h \rightarrow 0$, and near the chiral phase transition the dynamical quark mass, which is proportional to the order parameter, is very small. Consequently, the thermodynamics of the QM model can be formulated as an effective theory for the order parameter. Due to the smallness of the quark mass the high temperature expansion can be applied to derive the effective quark potential [23 25]

$$
\begin{aligned}
& \Omega_{q \bar{q}}^{\mathrm{th}} \simeq N_{c} N_{f} T^{4}\left\{-\frac{7 \pi^{2}}{180}+\frac{1}{12} \frac{m_{q}^{2}}{T^{2}}+\frac{1}{8 \pi^{2}} \frac{m_{q}^{4}}{T^{4}} \times\right. \\
& \left.\left[\ln \left(\frac{m_{q}}{\pi T}\right)+\gamma_{E}-\frac{3}{4}\right]+\frac{7 \zeta(3)}{192 \pi^{4}} \frac{m_{q}^{6}}{T^{6}}+\mathcal{O}\left(\frac{m_{q}^{8}}{T^{8}}\right)\right\},
\end{aligned}
$$

where $\gamma_{E}$ is Euler's constant. Note that there is no cubic quark mass term in this expansion in contrast to the bosonic case.

The effective potential of the QM model is the sum of the quark and meson contributions

$$
\Omega(\sigma)=\Omega_{q \bar{q}}^{\mathrm{th}}-\frac{\lambda v^{2}}{2} \sigma^{2}+\frac{\lambda}{4} \sigma^{4} .
$$

We note that for $\lambda>0$, one would expect the phase transition to be second order.

Because the dynamical quark mass is proportional to the order parameter $\sigma$, the logarithmic term in Eq. (15) competes with the quartic term in the meson potential (16). For sufficiently small values of the order parameter, the logarithmic term is arbitrarily large and negative. This leads to a change in sign of the effective quartic coupling in Eq. (16), which implies that the phase transition is first order for any finite value of $\lambda$. This effect resembles the Coleman-Weinberg fluctuation-induced firstorder phase transition [26] at $T=0$. In order to make the mechanism more transparent, we introduce the Landau effective potential, corresponding to Eq. (16)

$$
\Omega(\sigma)-\Omega_{\mathrm{bg}}=\frac{1}{2} a(T) \sigma^{2}+\frac{1}{4} b \sigma^{4}\left[1+c \ln \left(\sigma / \sigma_{0}\right)\right],
$$

where, to linear order in $T-T_{c}, a(T)=A \cdot\left(T-T_{c}\right)$ and $A>0$. The quartic coupling $b$ as well as the coefficient $c$ are assumed to be positive constants. In general, both $b$ and $c$ depend on temperature. However, this dependence is irrelevant for our discussion, as long as $b\left(T_{c}\right)$ and $c\left(T_{c}\right)$ are non-zero.

The parameters of the Landau potential (17) can be related to that of the QM model. By inspection of Eq. (15) and (16) one finds

$$
\begin{aligned}
a & =\frac{g^{2} N_{c} N_{f}}{6}\left(T^{2}-T_{c}^{2}\right) \\
& =A \cdot\left(T-T_{c}\right)+\mathcal{O}\left[\left(T-T_{c}\right)^{2}\right],
\end{aligned}
$$

where

$$
\begin{aligned}
T_{c}^{2} & =\frac{6 \lambda v^{2}}{g^{2} N_{c} N_{f}}, \\
A & =g^{2} N_{c} N_{f} T_{c} / 3 .
\end{aligned}
$$

Similarly, one can identify the quartic coupling $b$ and the coefficient $c$

$$
\begin{aligned}
& b=\lambda+\frac{g^{4} N_{c} N_{f}}{2 \pi^{2}}\left(\gamma_{E}-\frac{3}{4}\right), \\
& c=\frac{g^{4} N_{c} N_{f}}{2 \pi^{2} b},
\end{aligned}
$$

as well as the parameter $\sigma_{0}$, which for $T \simeq T_{c}$ is given by

$$
\sigma_{0}=\frac{\pi T}{g} \approx \frac{\pi T_{c}}{g} .
$$



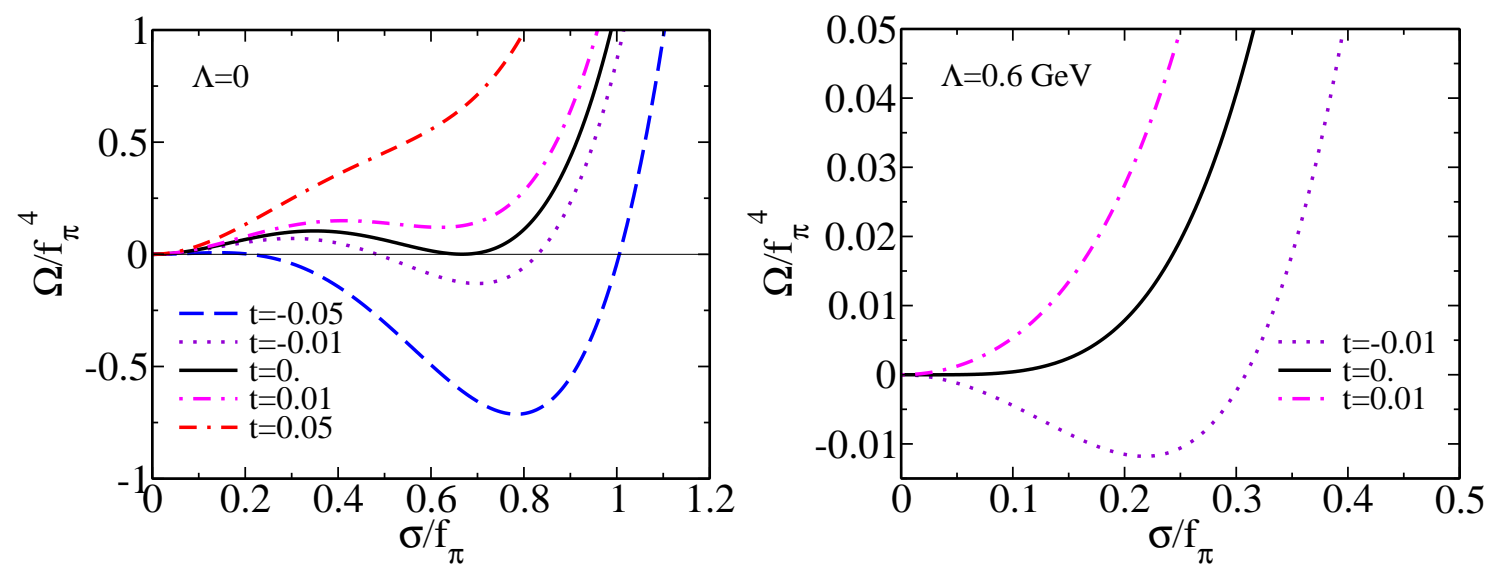

FIG. 1: The effective potential as a function of the order parameter in the vicinity of the phase transition for vanishing (left panel) and finite (right panel) cutoffs. The curves correspond to $\mu=0$ and a few temperature close to $T_{P T}$. The potential is normalized to zero at the origin.

The background contribution $\Omega_{\mathrm{bg}}$ in Eq. (17) is independent of the order parameter $\sigma$, and given by the $T^{4}$ term in Eq. (15).

For small positive values of $a$, i.e. for $T \gtrsim T_{c}$, the potential (17) has two minima located at

$$
\begin{aligned}
& \sigma_{1} / \sigma_{0}=0, \\
& \sigma_{2} / \sigma_{0}=\kappa-\frac{a}{2 b c \kappa \sigma_{0}^{2}},
\end{aligned}
$$

where $\kappa=\exp (-1 / 4-1 / c)$. At these points, the corresponding values of the potential are

$$
\begin{aligned}
& \Omega\left(\sigma_{1}\right)-\Omega_{\mathrm{bg}}=0 \\
& \Omega\left(\sigma_{2}\right)-\Omega_{\mathrm{bg}}=-\frac{1}{4} b c\left(\sigma_{0} \kappa\right)^{4}+a\left(\sigma_{0} \kappa\right)^{2} .
\end{aligned}
$$

Consequently, for $a<\frac{1}{4} b c\left(\sigma_{0} \kappa\right)^{2}$ the second solution, $\sigma_{2}$, is energetically favored. For $a=\frac{1}{4} b c\left(\sigma_{0} \kappa\right)^{2}$ the two minima at $\sigma_{1}=0$ and $\sigma_{2}=\frac{3}{4} \kappa \sigma_{0}$ are degenerate, i.e. $\Omega\left(\sigma_{1}\right)=\Omega\left(\sigma_{2}\right)=\Omega_{\mathrm{bg}}$. Finally, for $a>\frac{1}{4} b c\left(\sigma_{0} \kappa\right)^{2}$ the first solution $\sigma_{1}$ is energetically favored. This analysis indicates that a first-order phase transition occurs at $T_{P T}=T_{c}+b c\left(\kappa \sigma_{0}\right)^{2} /(4 A)$, i.e. at a temperature above the nominal critical temperature $T_{c}$.

The results obtained above are illustrated in Fig. 1-left, where we show the effective potential of the QM model for $\mu=0$, neglecting the fermion vacuum term. The dependence of the potential on the sigma field, for selected values of the reduced temperature $t=\left(T-T_{P T}\right) / T_{P T}$, shows explicitly that this model exhibits a first-order phase transition in the chiral limit [33]. Thus, without the fermion vacuum term, this model does not reproduce the second-order transition of QCD with two massless flavors, expected from the universality argument.

\section{B. Effective potential with the fermion vacuum contribution}

We now include the contribution of the fermion vacuum term of the QM model and analyze the resulting thermodynamic potential. For sufficiently small values of the dynamical quark mass $m_{q}$, the vacuum term in Eq. (9) can be expanded in a Puiseux series in $m_{q} / \Lambda$. The leading terms of this expansion reads

$$
\begin{aligned}
& \Omega_{q \bar{q}}^{\mathrm{vac}}=-2 N_{f} N_{c} \int \frac{d^{3} p}{(2 \pi)^{3}} E_{q} \theta\left(\Lambda^{2}-\vec{p}^{2}\right) \simeq \\
& -\frac{N_{f} N_{c} \Lambda^{4}}{4 \pi^{2}}\left\{1+\frac{m_{q}^{2}}{\Lambda^{2}}+\frac{1}{8} \frac{m_{q}^{4}}{\Lambda^{4}}\left[1+4 \ln \left(\frac{m_{q}}{2 \Lambda}\right)\right]\right. \\
& \left.-\frac{1}{8} \frac{m_{q}^{6}}{\Lambda^{6}}+\mathcal{O}\left(\frac{m_{q}^{8}}{\Lambda^{8}}\right)\right\} .
\end{aligned}
$$

The logarithmic dependence on $m_{q}$ cancels between the thermal and vacuum contributions to the thermodynamic potential, (15) and (28). Consequently, when the fermion vacuum term is included, the effective potential has the typical structure expected for a theory with the secondorder phase transition

$$
\Omega(\sigma)-\Omega_{\mathrm{bg}}=\frac{1}{2} a(T) \sigma^{2}+\frac{1}{4} b \sigma^{4} .
$$

The mass coefficient is formally given by the Eq. (18), however with the following redefinition of the critical temperature

$$
T_{c}^{2}=\frac{3}{\pi^{2}} \Lambda^{2}+\frac{6 \lambda v^{2}}{g^{2} N_{c} N_{f}}
$$

and the quartic coupling reads

$$
b=\lambda+\frac{g^{4} N_{c} N_{f}}{2 \pi^{2}}\left[\gamma_{E}-1+\ln \left(\frac{2 \Lambda}{\pi T_{c}}\right)\right] .
$$


In the above expressions the parameters $\lambda$ and $v^{2}$ depend on the cutoff $\Lambda$, because we require that the physical vacuum parameters discussed above should be reproduced independently of the cutoff. Taking this dependence into account, the leading $\Lambda^{2}$ term in Eq. (30) is canceled by a corresponding term in $\lambda v^{2}$. Consequently, for large $\Lambda$, the critical temperature is cutoff independent:

$$
T_{c}^{2}\left(\Lambda \gg T_{c}\right)=\frac{3 m_{\sigma}^{2} f_{\pi}^{2}}{N_{c} N_{f}\left(m_{q}^{0}\right)^{2}}+\frac{3\left(m_{q}^{0}\right)^{2}}{2 \pi^{2}}+\mathcal{O}\left(\Lambda^{-2}\right) .
$$

The quartic coupling is not positive definite for all values of $\Lambda$. For $\Lambda>\Lambda^{*}$, where $\Lambda^{*}$ is defined by $b\left(T \rightarrow T_{c}, \Lambda^{*}\right)=0$, the thermodynamic potential (29) has only one minimum at any temperature, at $\sigma_{1}=0$ or at $\sigma_{2}=\sqrt{-a / b}$. Thus, a second-order phase transition takes place at $T_{P T}=T_{c}$. This behavior is illustrated in Fig. 1-right, where we show the thermodynamic potential obtained in the QM model, including the fermion vacuum term, for $\Lambda=0.6 \mathrm{GeV}$, which implies $\lambda=3.06$ and $v^{2}=-(578)^{2} \mathrm{MeV}^{2}$. For this choice of parameters, the position of the minimum of the thermodynamic potential varies continuously with temperature, indicating a second-order phase transition.

However, for $\Lambda<\Lambda^{*}$, the quartic coupling is negative, while, as shown in Eqs. (15) and (28), the coefficient of the $\sigma^{6}$ term is always positive, as required for stability. The negative value of $b$ results in the appearance of the second minimum in the potential, leading to the firstorder phase transition. This is similar to the situation described in the previous section, where the fermion vacuum term was neglected. The appearance of a first-order phase transition for small $\Lambda$ is illustrated in Fig. 2 where the temperature dependence of the order parameter is shown for several values of the cutoff $\Lambda$. For $\Lambda<0.3$ $\mathrm{GeV}$, the order parameter exhibits hysteresis, characteristic of a first-order phase transition. For the parameter set employed here, we find $\Lambda^{*} \simeq 244 \mathrm{MeV}$. This value is comparable to the physical scales that characterizes

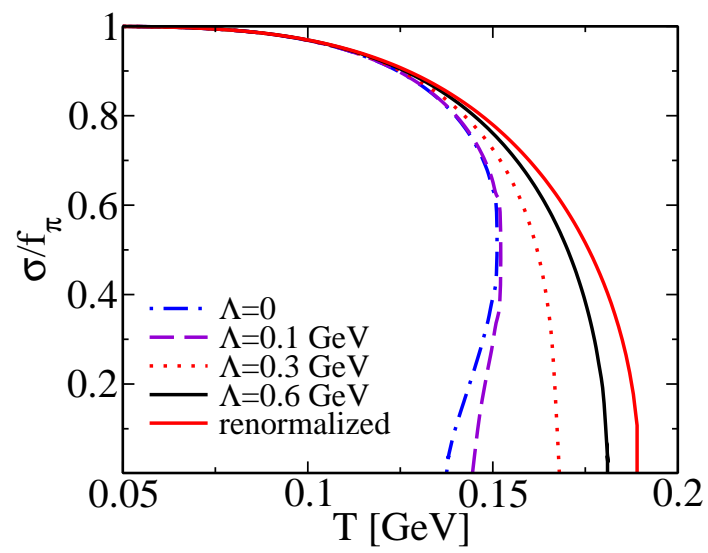

FIG. 2: The order parameter as a function of temperature for different values of the cutoff parameters. The uppermost line corresponds to the renormalized theory. the vacuum. In general, the cutoff should be larger than any physical scale of a system. In this case we find a second-order chiral phase transition in the QM model.

The fermion vacuum fluctuations modify the critical properties of the QM model considerably. The modification depends on the value of the ultraviolet cutoff $\Lambda$, which is introduced to regularize the divergent vacuum term. For large cutoffs $\left(\Lambda>\Lambda^{*}\right)$, the QM model exhibits a second-order phase transition, whereas in the opposite limit the phase transition stays first order, as in the approximation, where the vacuum term is completely neglected.

Clearly, a finite cutoff modifies not only the critical structure of the medium but also the properties of physical observables. Hence, it is desirable to remove the cutoff dependence by means of a suitable renormalization procedure. This problem will be addressed in the next section.

\section{RENORMALIZATION OF THE FERMION VACUUM TERM}

The expansion (28) indicates that, in addition to the fermion vacuum term, also the mass parameter $a$ and the quartic coupling $b$ diverge in the limit of $\Lambda \rightarrow \infty$. These divergencies can be regularized by introducing a cutoff, as done above. In this section we choose a more convenient route and use the dimensional regularization scheme.

The vacuum term is, to lowest order, just the one-loop effective potential at zero temperature 24]

$$
\begin{aligned}
\Omega_{q \bar{q}}^{\mathrm{vac}} & =-2 N_{f} N_{c} \int \frac{d^{3} p}{(2 \pi)^{3}} E_{q} \\
& =-2 N_{f} N_{c} \int \frac{d^{4} p}{(2 \pi)^{4}} \ln \left(p_{0}^{2}+E_{q}^{2}\right)+\mathrm{C},
\end{aligned}
$$

where the infinite constant $C$ is dropped, since it is independent of the fermion mass.

To regularize Eq. (33) we perform the dimensional regularization near three dimensions, $d=3-2 \epsilon$. The resulting potential up to zeroth order in $\epsilon$ reads

$$
\Omega_{q \bar{q}}^{\mathrm{vac}}=\frac{N_{c} N_{f}}{16 \pi^{2}} m_{q}^{4}\left\{\frac{1}{\epsilon}-\frac{1}{2}\left[-3+2 \gamma_{E}+4 \ln \left(\frac{m_{q}}{2 \sqrt{\pi} M}\right)\right]\right\},
$$

where $M$ is an arbitrary renormalization scale parameter.

The thermodynamic potential is then renormalized by adding a counter term to the Lagrangian of the QM or PQM model. With the choice 34]

$$
\delta \mathcal{L}=\frac{N_{c} N_{f}}{16 \pi^{2}} g^{4} \sigma^{4}\left\{\frac{1}{\epsilon}-\frac{1}{2}\left[-3+2 \gamma_{E}-4 \ln (2 \sqrt{\pi})\right]\right\},
$$

the renormalized contribution of the fermion vacuum loop reads

$$
\Omega_{q \bar{q}}^{\mathrm{reg}}=-\frac{N_{c} N_{f}}{8 \pi^{2}} m_{q}^{4} \ln \left(\frac{m_{q}}{M}\right) .
$$


The quark contribution to the thermodynamic potential is then given by Eq. (9), with the vacuum term replaced by Eq. (36).

For large values of the order parameter, $m_{q}=g \sigma>M$, the renormalized vacuum contribution in Eq. (36) is unbounded from below. At $T=0$, the potential (8), including the renormalized quark vacuum term, has a minimum at the physical point $\sigma=f_{\pi}$, a maximum at $\sigma \simeq 212$ $\mathrm{MeV}$ and then decreases monotonously to $-\infty$ for $\sigma \rightarrow \infty$ [35]. The instability for large $\sigma$ is symptomatic of the renormalized one-loop approximation [26].The inclusion of higher order loop contributions is known to cure this problem.

Dimensional regularization introduces an arbitrary renormalization scale parameter $M$. Consequently, the parameters $v$ and $\lambda$ are $M$ dependent through the conditions (13) and (12). Nevertheless, in the one-loop approximation, physical quantities are independent of $M[26$. Indeed, it is rather straightforward to show that

$$
\frac{d \Omega_{\mathrm{MF}}\left(\lambda[M], v^{2}[M], M\right)}{d M}=0 .
$$

Sufficiently close to the phase transition, the Landau potential corresponding to the renormalized QM model has the form (29), with the mass parameter given by Eqs. (18) 20). The critical temperature $T_{c}$ is independent of the renormalization scale, since $\partial\left(\lambda v^{2}\right) / \partial M=0$. Consequently, it can be expressed solely in terms of physical quantities,

$$
T_{c}^{2}=\frac{3 m_{\sigma}^{2} f_{\pi}^{2}}{N_{c} N_{f}\left(m_{q}^{0}\right)^{2}}+\frac{3\left(m_{q}^{0}\right)^{2}}{2 \pi^{2}} .
$$

This expression for $T_{c}$ agrees with the one obtained in the cutoff regularization scheme, Eq. (32), for $\Lambda \rightarrow \infty$. Also the quartic coupling $b$ can be expressed in terms of physical quantities

$$
b=\frac{m_{\sigma}^{2}}{2 f_{\pi}^{2}}+\frac{g^{4} N_{c} N_{f}}{2 \pi^{2}}\left[\gamma_{E}+\ln \left(\frac{g f_{\pi}}{\pi T_{c}}\right)\right] .
$$

Thus, we conclude that in the mean-field approximation, the thermodynamic potential of the quark-meson model, including the renormalized fermionic vacuum contribution, is uniquely defined. In Fig. 2 we show the temperature dependence of the order parameter, as obtained within this model. This and other physical quantities are, as indicated above, free from ambiguities related to the choice of the renormalization scale. In the chiral limit, this model exhibits a second-order phase transition at $\mu=0$, as expected for two-flavor QCD. Thus, the quark-meson model, with appropriately renormalized fermionic vacuum fluctuations, is an effective QCD-like model, suitable for describing the physics at the chiral phase transition.

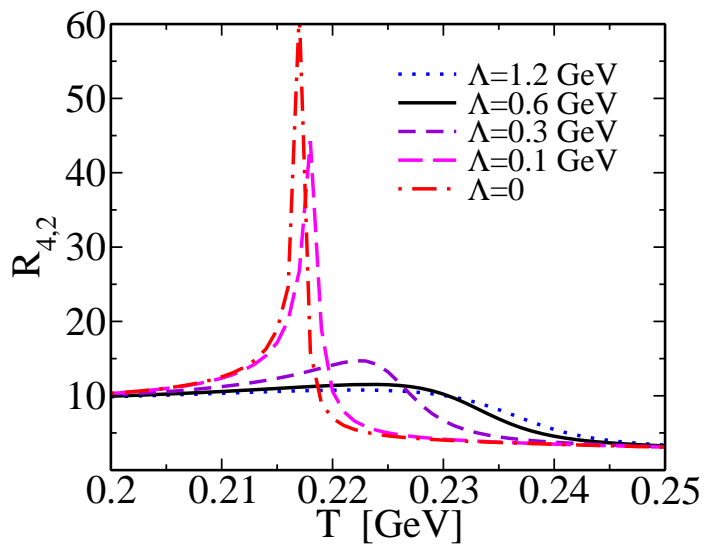

FIG. 3: The kurtosis in the PQM model as a function of temperature for several values of cutoff parameters $\Lambda$.

\section{QUARK NUMBER DENSITY FLUCTUATIONS}

As discussed above, the fermion vacuum term is required to obtain the correct critical behavior in the chiral limit. In this section, we show that the vacuum term is important also for the thermodynamics for non-zero pion masses. To illustrate this, we consider fluctuations of the net quark number density within the PQM model.

The fluctuations of the quark number density are characterized by the generalized susceptibilities,

$$
c_{n}(T)=\left.\frac{\partial^{n}\left[p(T, \mu) / T^{4}\right]}{\partial(\mu / T)^{n}}\right|_{\mu=0} .
$$

The first two coeffients, $c_{2}$ and $c_{4}$, are the second and the fourth cumulants of the net quark number $N_{q}$,

$$
\begin{aligned}
& c_{2}=\frac{\chi_{q}}{T^{2}}=\left\langle\left(\delta N_{q}\right)^{2}\right\rangle, \\
& c_{4}=\left\langle\left(\delta N_{q}\right)^{4}\right\rangle-3\left\langle\left(\delta N_{q}\right)^{2}\right\rangle^{2} .
\end{aligned}
$$

Here $\chi_{q}$ is the quark number susceptibility and $\delta N_{q}=$ $N_{q}-\left\langle N_{q}\right\rangle$.

The coefficients $c_{n}(T)$ are sensitive probes of the chiral phase transition, see e.g. [29]. In particular, $c_{2}$ diverges at the critical endpoint of the QCD phase diagram [2]. In the chiral limit and at non-zero chemical potential, all generalized susceptibilities $c_{n}(T)$ with $n>2$ diverge at the $O(4)$ chiral critical line [30]. Moreover, if the spinodal instability is reached, fluctuations of the net quark number also diverge at a first-order chiral phase transition [4].

A very particular role is attributed to the so called kurtosis of the net quark number fluctuations [8, 28, 30, 31], the ratio of $c_{4}$ and $c_{2}$

$$
R_{4,2}=\frac{c_{4}}{c_{2}}
$$

This observable is not only sensitive to the chiral phase transition but also probes the confinementdeconfinement transition. At very low and very high 

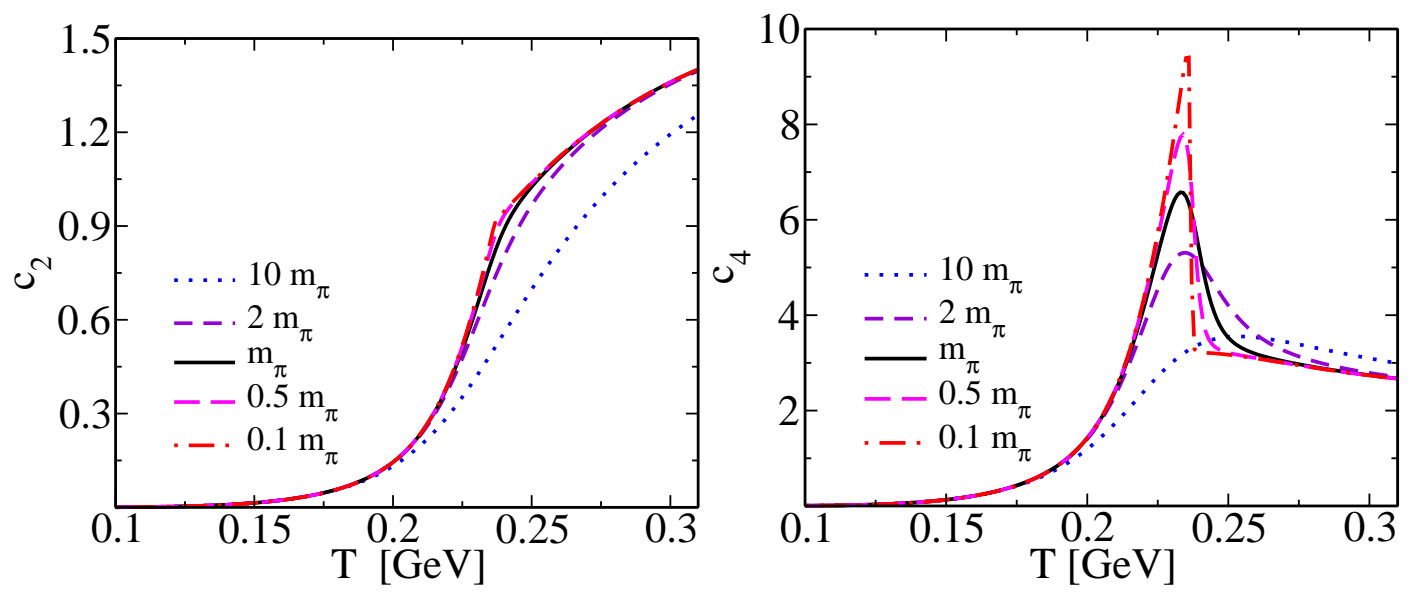

FIG. 4: The second $c_{2}$ and the fourth $c_{4}$ order cumulants, computed in the PQM model including the renormalized fermion vacuum potential, as functions of temperature for several values of the pion mass.

temperatures, the kurtosis reflects the quark content of the baryon-number carrying effective degrees of freedom [30, 31]. Thus, at low temperatures, in the confined phase, $R_{4,2} \simeq N_{q}^{2}=9$ while in an ideal gas of quarks [36] $R_{4,2} \sim 1$. The influence of the chiral phase transition on the kurtosis has been studied within lattice gauge theory [32]. Due to the "statistical confinement" realized in the PQM and PNJL models, these models can also be used to explore the interplay between confinement and chiral restoration e.g. in the kurtosis [8, 31].

We first consider the dependence of the ratio $R_{4,2}$ on the cutoff $\Lambda$ in the PQM model, including the vacuum contribution, Eq. (28). In Fig. 3 the kurtosis is shown at the physical pion mass, as a function of the temperature for several values of $\Lambda$. The kurtosis converges to $R_{4,2}=9$ at low temperatures and to $R_{4,2} \sim 1$ in the high temperature limit for all values of cutoff parameter. However, near $T_{c}$ the kurtosis is very sensitive to $\Lambda$; for small cutoffs it develops a peak, which is maximal for $\Lambda \rightarrow 0$. The sharp peak, obtained for vanishing cutoff, is a consequence of the underlying first-order phase transition in the chiral limit. Clearly, such a strong dependence on the ultraviolet cutoff is unphysical and must be removed by a suitable renormalization of the vacuum term.

Having established the relevance of the fermion vacuum term for the kurtosis, we now study fluctuations of the net quark density in the PQM model, including the renormalized vacuum contribution. In Fig. 4 we show the second and fourth cumulants, $c_{2}$ and $c_{4}$, as functions of temperature for several values of the pion mass. For small $m_{\pi}, c_{2}$ exhibits a kink at the chiral crossover transition, while $c_{4}$ shows an rapid drop, which in the chiral limit evolves into a discontinuity.

These results can be understood in Landau theory. For small $\sigma$, the thermodynamic potential reads

$$
\Omega(T, \mu)-\Omega_{b g}(T, \mu)=\frac{1}{2} a(T, \mu) \sigma^{2}+\frac{1}{4} b \sigma^{4}-h \sigma,
$$

where $\Omega_{b g}(T, \mu)$ is the background (regular) contribution, which is independent of $\sigma$. The external field $h$ breaks the symmetry and generates a finite pion mass. For a small $\mu$ and $T$ near the chiral transition temperature, the coefficient $a(T, \mu)$ can be parameterized by [30]

$$
a(T, \mu)=A \frac{T-T_{c}}{T_{c}}+B\left(\frac{\mu}{T_{c}}\right)^{2}
$$

where both, $A$ and $B$ are positive constants. The coefficients of the effective potential can be expressed in terms of the parameters of the (P)QM model by considering the expansion of the thermodynamic potential from Eq. (8) for small $m_{q}$ and $\sigma$.

In the chiral limit and at $\mu=0$ the singular contribution to the second and fourth cumulants is

$$
\begin{aligned}
c_{2}^{\text {sing }} & =\frac{A B}{b T_{c}^{4}} \frac{T-T_{c}}{T_{c}} \theta\left(T_{c}-T\right), \\
c_{4}^{\text {sing }} & =\frac{6 B^{2}}{b T_{c}^{4}} \theta\left(T_{c}-T\right) .
\end{aligned}
$$

Hence, $c_{2}$ has a kink at the critical point, while $c_{4}$ is discontinuous, in agreement with the numerical results for the PQM model presented above.

The peak structure in $c_{4}$ near the chiral transition, shown in Fig. 4, results from an interplay of the regular part, which increases as the critical point is approached, and the discontinuity of the singular part.

The dependence of the fourth cumulant $c_{4}$ on the pion mass is also reflected in the kurtosis of the quark number fluctuations. In Fig. 5 we show $R_{4,2}$ in the vicinity of the chiral phase transition, for several values of the pion mass. The transition between the low and high temperature regimes is sharpened as the pion mass is lowered, indicating that this observable is sensitive not only to deconfinement but also to chiral dynamics. In the meanfield approximation, a clear enhancement beyond the low temperature value, $R_{4,2}=9$, is found just below the pseudo-critical temperature, at the physical value of the 
pion mass. There is a further increase of the enhancement when approaching the chiral limit, where $R_{4,2}$ exhibits a discontinuity at the chiral phase transition, in agreement with Eq. (47). However, when the renormalized vacuum term is included, this dependence is weaker than previously obtained within the PQM model, neglecting vacuum fluctuations [31]. This shows, that an appropriate renormalization of the fermionic vacuum potential is essential for a consistent formulation of the thermodynamics of the PQM model.

\section{SUMMARY AND CONCLUSIONS}

We have discussed the importance of the divergent fermion vacuum potential in a consistent formulation of the thermodynamics of the quark-meson (QM) and the Polyakov loop extended quark-meson (PQM) models.

We have shown that the vacuum term influences the order of the chiral phase transition in the chiral limit. The QM and PQM models exhibit a first-order phase transition at $\mu=0$, when the fermion vacuum term is neglected. On the other hand, when fermion vacuum fluctuations are included, a second-order phase transition of the $\mathrm{O}(4)$ universality class is possible, as expected in models that exhibit the $S U(2) \times S U(2)$ chiral symmetry.

Also for the physical value of the pion mass, the vacuum term influences thermodynamic observables near the chiral crossover transition. We have illustrated this explicitly, by computing the second and the fourth cumulants as well as the kurtosis of the net quark number fluctuations for several values of the pion mass using different regularization schemes for the fermion vacuum potential.
Finally, we have renormalized the fermion vacuum term to obtain the thermodynamic potentials of the QM and PQM models, free of any dependence on the unphysical ultraviolet cutoff.

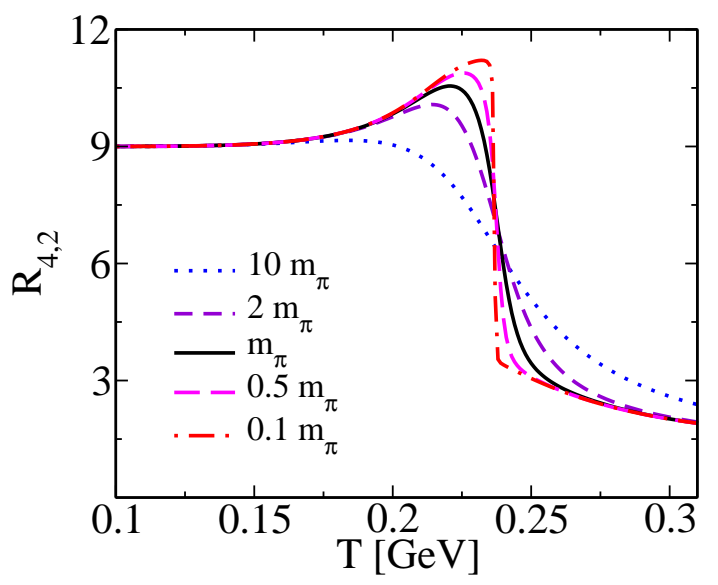

FIG. 5: The kurtosis $R_{4,2}$ as a function of temperature for several values of the pion mass in the PQM model, including the renormalized fermion vacuum term.

\section{Acknowledgments}

We acknowledge stimulating discussions with Yu. Ivanov, E. Kolomeitsev and V. Pangon. E. Nakano and V. Skokov acknowledge the financial support by the Frankfurt Institute for Advanced Studies (FIAS). K. Redlich acknowledges discussions with H. Fujii and C. Sasaki and partial supports from the Polish Ministry of Science (MEN) and the Alexander von Humboldt Foundation (AvH).
[1] A. Bazavov et al., Phys. Rev. D 80, 014504 (2009); S. Ejiri et al., Phys.Rev.D 80, 094505 (2009); C.R. Allton et al., Phys. Rev. D 71, 054508 (2005); Y. Aoki et al., JHEP 0906, 088 (2009); Z. Fodor and S.D. Katz, Nucl. Phys. News 16N3, 12 (2006).

[2] M. A. Stephanov, PoS LAT2006, 024 (2006).

[3] For a review see e.g., S. P. Klevansky, Rev. Mod. Phys. 64, 649 (1992).

[4] C. Sasaki, B. Friman, and K. Redlich, Phys. Rev. D 75, 054026 (2007); Phys. Rev. D 77, 034024 (2008); Phys. Rev. Lett. 99, 232301 (2007).

[5] K. Fukushima, Phys. Lett. B 591, 277 (2004).

[6] C. Ratti, M. A. Thaler and W. Weise, Phys. Rev. D 73, 014019 (2006); C. Sasaki, B. Friman, and K. Redlich, Phys. Rev. D 75, 074013 (2007); K. Fukushima, J. Phys. G 36, 064020 (2009); Phys. Rev. D 79, 074015 (2009); T. Hell, M. Cristoforetti, and W. Weise, Phys. Rev. D 79, 014022 (2009); M. Cristoforetti, T. Hell, B. Klein, and W. Weise, e-Print: arXiv:1002.2336 [hep-ph].

[7] B.-J. Schaefer, J. M. Pawlowski and J. Wambach, Phys. Rev. D 76, 074023 (2007).

[8] B.-J. Schaefer, M. Wagner, J. Wambach, Phys. Rev. D
81, 074013 (2010).

[9] B.-J. Schaefer, M. Wagner, Phys. Rev. D 79, 014018 (2009).

[10] O. Scavenius, A. Mocsy, I. N. Mishustin and D. H. Rischke, Phys. Rev. C 64, 045202 (2001).

[11] R. Friedberg and T. D. Lee, Phys. Rev. D 15, 1694 (1977); Phys. Rev. D 16, 1096 (1977); Phys. Rev. D 18, 2623 (1978); B.-J. Schaefer and J. Wambach, Nucl. Phys. A 757, 479 (2005); B.-J. Schaefer, H.-J. Pirner, Nucl. Phys. A660 , 439 (1999).

[12] B.-J. Schaefer and J. Wambach, Phys. Rev. D 75, 085015 (2007).

[13] E. S. Bowman and J. I. Kapusta, Phys. Rev. C 79, 015202 (2009).

[14] U. S. Gupta and V. K. Tiwari, arXiv:0911.2464 [hep-ph].

[15] D. Nickel, Phys. Rev. D 80, 074025 (2009).

[16] T. Kahara and K. Tuominen, Phys. Rev. D 80, 114022 (2009).

[17] J. I. Kapusta and E. S. Bowman, Nucl. Phys. A 830, $721 \mathrm{C}(2009)$.

[18] E. Nakano, B.-J. Schaefer, B. Stokic, B. Friman and K. Redlich, Phys. Lett. B 682, 401 (2010). 
[19] H. Fujii and M. Ohtani, Phys. Rev. D 70, 014016 (2004).

[20] A. J. Mizher, M. N. Chernodub and E. S. Fraga, arXiv:1004.2712 [hep-ph].

[21] R. D. Pisarski and F. Wilczek, Phys. Rev. D 29, 338 (1984).

[22] J. Gasser and H. Leutwyler, Nucl. Phys. B 250, 465 (1985).

[23] L. Dolan and R. Jackiw, Phys. Rev. D 9, 3320 (1974).

[24] M. Quiros, arXiv:hep-ph/9901312

[25] N. P. Landsman and C. G. van Weert, Phys. Rept. 145, 141 (1987).

[26] S. Coleman and E. Weinberg, Phys. Rev. D 7, 1888 (1973).

[27] C. R. Allton et al., Phys. Rev. D 68, 014507 (2003).

[28] F. Karsch, S. Ejiri and K. Redlich, Nucl. Phys. A 774, 619 (2006); S. Ejiri et al., Nucl. Phys. A 774, 837 (2006).

[29] M. Wagner, A. Walther, B.-J. Schaefer, Comput. Phys.
Commun. 181, 756 (2010); B.-J. Schaefer, M. Wagner, J. Wambach, PoS CPOD 2009, 017 (2009), arXiv:0909.0289 [hep-ph].

[30] S. Ejiri, F. Karsch and K. Redlich, Phys. Lett. B 633, 275 (2006).

[31] B. Stokic, B. Friman and K. Redlich, Phys. Lett. B 673, 192 (2009).

[32] M. Cheng et al., Phys. Rev. D 79, 074505 (2009).

[33] Note that here the transition temperature $T_{P T}$ is not given by the analytical expression given above, but is determined numerically within the QM model.

[34] Here we use the freedom in the definition of counter terms to remove irrelevant constants.

[35] We note that for finite $\Lambda$, the cutoff regularized potential, Eq. (28), is always bounded from below.

[36] More precisely, this number is $6 / \pi^{2}$ due to quantum statistics. 